\title{
Back Reaction from Trace Anomaly in RN-blackholes Evaporation*
}

\author{
F. Canfora and G. Vilasi ${ }^{\dagger}$ \\ Istituto Nazionale di Fisica Nucleare, GC di Salerno, Italy. \\ Dipartimento di Fisica "E.R.Caianiello", Università di Salerno.
}

\begin{abstract}
A model is proposed to describe a transition from a charged black hole of mass $M$ and charge $Q$ to one of mass $\bar{M}$ and charge $\bar{Q}$. The basic equations are derived from the non-vacuum Einstein field equations sourced by the Coulomb field and by a null scalar field with a nonvanishing trace anomaly. It is shown that the nonvanishing trace of the energy-momentum tensor prevents the formation of a naked singularity.
\end{abstract}

KEYWORDS: Blackhole evaporation, Back-reaction, QFT Unitarity.

* published in JHEP12(2003)055

†Corresponding author: Gaetano Vilasi, Università di Salerno, Dipartimento di Fisica "E.R.Caianiello", Via S.Allende, 84081 Baronissi (Salerno) Italy; e-mail: vilasi@sa.infn.it, Phone: +39-089-965317, Fax: $+39-089-965275$. 


\section{Contents}

1. The model 2

2. The Einstein tensor 3

3. The source 1

4. The Einstein equations 6

5. The boundary conditions 8

6. The non vanishing final mass 9

7. Field-theoretical features 12

8. Conclusions and perspectives 14

One of the most outstanding results of Quantum Field Theory (QFT) on curved spacetime is the Hawking radiation discovery [22] which strengthened the analogy between blackhole mechanics and thermodynamics [2]. Unfortunately, since a complete evaporation would violate unitarity, the consequences of the Hawking radiation seem to undermine the physical basis either of QFT or of the general relativity itself. Indeed, the Hawking particles, and, more generally, quantum sources violating the energy conditions, are able to violate the third law of blackhole thermodynamics, eventually giving rise to naked singularities. Recently, the role of the four dimensional trace anomaly in the blackhole evaporation has been investigated [16]. Here, we want to analyze the effects of Hawking particles and of trace anomaly on another important feature of blackhole thermodynamics: the third law.

The third law, analogue of the third law of thermodynamics, states that, if the energymomentum tensor of matter sources satisfies the weak energy condition, then the surface gravity of the blackhole, that plays the role of the temperature, cannot be lowered down to zero in a finite series of steps 24]. This is not the case for energy-momentum tensors arising as vacuum expectation values of quantum fields, as in the Hawking radiation. Therefore, the third law can be violated during the evaporation and, more generally, when energymomentum tensors not satisfying positivity conditions come into play. This could have important consequences also for another of main unsolved problems in general relativity: the cosmic censorship hypothesis $(\mathrm{CCH})$ which, roughly speaking, states that singularities are always hidden behind a horizon. Indeed, if a suitable quantum field is able to lower the surface gravity of a blackhole down to zero in a finite time, we could end up with a naked singularity. This could be the case during the evaporation of a charged blackhole 
if one suppose that Hawking particles carry away mass but not charge to infinity. This supposition is not unlike since the Hawking particles are massless (the emission of massive particles is highly suppressed) while charged massless particles do not exist. Despite many attempts [26] $\mathrm{CCH}$ is still unproved. Furthermore, some beautiful exact results in the spherically symmetric cases [15], [17] show that it is possible to construct naked singularity even with reasonable matter sources, this being an indication of the fact that $\mathrm{CCH}$ could need quite stronger hypothesis than the third law to be proved in general. Some insights into the physics of $\mathrm{CCH}$ came from the analysis of particular cases in which explicit calculations can be carried on. In the interesting cases of charged blackholes, without or with cosmological constant [14], [12], the stability analysis showed that naked singularities are unstable against linear perturbations.

Thus, it is quite interesting to study the effects of quantum sources, such as the Hawking radiation, on the surface gravity of an initially "dressed" Reissner-Nordstrom blackhole. Here we propose a toy model to investigate the following question: is it possible to transform an initially non extremal charged blackhole into an extremal one by means of Hawking particles?

Indeed, the most promising candidates to lower the surface gravity are energy-momentum tensors arising as vacuum expectation values of quantum fields since such tensors are able to violate all the energy conditions ${ }^{1}$. Furthermore, they are not "strange" enough to be neglected, being quite generic in Quantum Field Theory (QFT). We will prove, via the exact Einstein equations, that, in this model, an energy-momentum tensor describing a null quantum fluid (that is, a null fluid violating all the energy conditions) with a nonvanishing trace anomaly cannot lower the surface gravity of the charged blackhole, so that the evaporation stops. Thus, the trace anomaly, that is a generic feature of QFT, could save the third law and prevent the formation of a naked singularity even if the energy-momentum tensor of the Hawking particles violates all the energy conditions.

\section{The model}

It is often claimed that blackholes evaporation can be explained only by a full quantum theory of gravity such as the superstring theory [18] or the loop quantum gravity [36]. Recently, more "conservative" approaches to the topic, such as the effective action [31, [] and the renormalization group method [9], also have given new insights into the physics of the evaporation. However, until the final stages, the evaporation is not a strong gravitational field phenomenon because the blackhole mass decreases slowly with time [3]. Accordingly, the standard Einstein equations with a suitable matter source can describe how the metric evolves during the evaporation. Moreover, dynamical implementations of loop quantum gravity show that discrete eigenvalues of geometric operators converge in a few Planck lengths to their semiclassical approximations [10] [11].

Thus, far away (with respect to the Planck length) from the horizon a semiclassical description should describe carefully the evaporation process. Therefore, this is an accurate

\footnotetext{
${ }^{1}$ Because of the Israel theorem [24], these violations of the energy conditions are necessary in order to violate the third law.
} 
tool for our purposes, since, to study the evaporation, one has to look at the mass of the blackhole brought at infinity by Hawking particles. Furthermore, it would be nice to solve the problems raised by the blackhole evaporation, such as the loss of unitarity, by only using general relativity and QFT.

The standard semiclassical program [3], [34], [40], [27], [35], [29], [30] to approach the analysis of the back-reaction of Hawking particles on the metric consists, first, in computing an effective vacuum stress-energy tensor of a null quantum field on the black hole space-time of interest [34] and, then, in using this tensor as a source for the Einstein equations [40], [27. In this framework, the results can be easily interpreted in terms of the unperturbed quantity, thus making manifest the changes of the blackhole space-time due to Hawking particles [40], 27].

However, it is quite hard to obtain the expression of the vacuum stress-energy tensor of quantum fields on a curved space-time. Usually, in four-dimensional geometries, it is possible to get approximated expressions for the energy-momentum tensor only in static situations [34], while, in dynamical models, such as the space-time of an evaporating blackhole, it is usually computed in effective two-dimensional geometries with the refined techniques available in string and conformal field theory [19], [25], [35], [32], [21] and in three-dimensional models with the powerful methods provided by three-dimensional gravity $^{2}$ [38], 39], [28]. Therefore, in such effective models, it is not possible to take into account the nontrivial effects of the four-dimensional trace anomaly.

Thus, a toy model is proposed in which it is possible to keep the form of the stressenergy tensor of quantum fields as general as possible and, at the same time, to fully take into account the trace of the stress-energy tensor of Hawking particles. It will be shown, via the exact Einstein equations, that this has important physical consequences.

\section{The Einstein tensor}

A transition from a Reissner-Nordstrom blackhole of initial mass $M$ and charge $Q$ to another one of final mass $\bar{M}$ and charge $\bar{Q}$ will be described outside the collapsed matter by means of a well suited source that carries away mass from the blackhole to infinity. The metric, valid only for $r>R^{*}>0, R^{*}$ denoting a radius larger than the initial radius of the outer horizon (and much larger than the Planck scale, as we will explain in more detail later on), will be written as in [3]:

$$
g=\left(1-\frac{m(r, v)}{r}\right) e^{2 j(r, v)} d v^{2}-2 e^{j(r, v)} d r d v-r^{2} d \Omega
$$

For $m=2 M-Q^{2} / r$ and $j=$ const, metric (2.1) reduces to the Reissner-Nordstrom metric in Eddington-Finkelstein coordinates.

\footnotetext{
${ }^{2}$ Some attempts to take into account the four-dimensional trace anomaly, in the framework of dilatonic gravity, can be found in [32].
} 
Since we want to probe the third law in the worst conditions, we will search for a solution of Einstein equations satisfying the following boundary conditions:

$$
\begin{aligned}
m(r, 0) & =2 M-\frac{Q^{2}}{r}, \quad M>|Q| ; \quad m(r, T)=2 \bar{M}-\frac{\bar{Q}^{2}}{r} \\
Q & =\bar{Q} ; \quad \bar{M} \geq 0 ; \quad j(r, 0)=k_{1} ; \quad j(r, T)=k_{2},
\end{aligned}
$$

where $k_{1}$ and $k_{2}$ are two constants and at $v=T$ the blackhole reaches its final state. In fact, by taking the final charge equal to the initial one $Q=\bar{Q}$, we compel the blackhole to loose its mass but not its charge so, in this way, by choosing a suitable energy-momentum tensor violating the weak energy condition, we can go as near as possible to the violation of the third law and, eventually, of the $\mathrm{CCH}$.

The above metric has the following scalar curvature

$$
\begin{aligned}
R & =\left\{-\exp (-j) 2 r^{2} \partial_{r v}^{2} j+3 r \partial_{r} j \partial_{r} m+m \partial_{r} j\right. \\
& +2 \partial_{r} m-4 r \partial_{r} j-2 r^{2}\left(1-\frac{m}{r}\right) \partial_{r}^{2} j \\
& \left.-2 r^{2}\left(1-\frac{m}{r}\right)\left(\partial_{r} j\right)^{2}+r \partial_{r}^{2} m\right\} \frac{1}{r^{2}}
\end{aligned}
$$

and the nonvanishing independent components of the Einstein tensor are

$$
\begin{aligned}
G_{v v} & =\frac{\exp (2 j)}{r^{3}}\left(m \partial_{r} m-r \exp (-j) \partial_{v} m-r \partial_{r} m\right) \\
G_{v r} & =\frac{\exp (j) \partial_{r} m}{r^{2}}, \quad G_{r r}=-2 \frac{\partial_{r} j}{r} \\
G_{\theta \theta} & =\frac{1}{2}\left\{-(2 r+m) \partial_{r} j-\exp (-j) 2 r^{2} \partial_{r v}^{2} j\right. \\
& +3 r \partial_{r} j \partial_{r} m-2 r^{2}\left(1-\frac{m}{r}\right) \partial_{r}^{2} j \\
& \left.-2 r^{2}\left(1-\frac{m}{r}\right)\left(\partial_{r} j\right)^{2}+r \partial_{r}^{2} m\right\} \\
G_{\varphi \varphi} & =\sin ^{2} \theta G_{\theta \theta} .
\end{aligned}
$$

\section{The source}

During an evaporation process, the black hole emits black-body radiation at the Hawking temperature. Since the emission of massive particles by the blackhole is strongly suppressed, the natural choice is to take as source, beyond the electromagnetic (e.m.) energy-momentum tensor $T_{\mu \nu}^{C}$, a null quantum fluid, so that

$$
T_{\mu \nu}=g_{\mu \nu} V(v, r)+\rho(v, r) u_{\mu} u_{\nu}+T_{\mu \nu}^{C},
$$

with $g(U, U)=0$, being $U$ the vector field naturally associated with the differential 1-form

$u_{\mu} d x^{\mu} \quad\left(u_{\mu}=g_{\mu \nu} U^{\nu}\right)$ and being $V$ the trace anomaly of quantum fields representing the Hawking particles. 
Thus, $T_{\mu \nu}$ has to be interpreted as the sum of the energy-momentum tensor of the e.m. field

$$
T_{\mu \nu}^{C}=g_{\mu \nu} \epsilon \frac{Q^{2}}{r^{4}},
$$

with $\epsilon=1$ for $\mu, \nu=1,2 ; \epsilon=-1$ for $\mu, \nu=3,4$, and a suitable average over quantum fields that contribute to the Hawking radiation and bring mass from the blackhole to infinity. However, we will be interested only in the asymptotic region $(r \rightarrow \infty)$ where Hawking particles, contributing to the black hole mass decrease, have to arrive. Thus, Coulomb terms which are of order $1 / r^{4}$ and then do not contribute to the Bondi mass-whose change we are interested in- can be neglected. We will take a neutral fluid as source because in such a way $T_{\mu \nu}$, bringing at infinity mass but not charge, allows in principle arbitrary violations of either the third law or the $\mathrm{CCH}$.

It is fair to say that as the Hawking temperature increases, the probability of charged particles production, specially electrons, increases as well. The inclusion of the corresponding black hole charge decrease is interesting but rather cumbersome because it is necessary to take into account the charge density continuity equation. In Section 6 , it will be shown that in a Q-constant process the trace anomaly is able to prevent the formation of a naked singularity. In its turn, the formation of a naked singularity is more difficult in a process in which the black hole charge decreases. This can be understood by comparing the distance $\left(\propto \sqrt{M^{2}-Q^{2}}\right)$ between the inner and the outer horizons in the two processes in which, without loss of generality, the mass variation may be assumed ${ }^{3}$ to be the same. In absence of numerical computations, we are still unable to predict the percentage of mass lost in a process in which the charge $\mathrm{Q}$ is allowed to decrease. At the moment, we only know that naked singularities do not occur and that all results also hold in the limit $Q \mapsto 0$, that is the trace anomaly stops the evaporation of a Schwarzschild black hole.

Since no constraints will be imposed on $\rho$ and $V$, the tensor $T_{\mu \nu}$ needs not to satisfy neither the weak nor the null energy conditions, thus providing us the violations we are searching for. To see this, let us write the weak energy condition:

$$
T_{\mu \nu} n^{\mu} n^{\nu} \geq 0
$$

for every non space-like future directed vector field $n^{\nu}$.

In the case of the energy-momentum tensor in Eq. (3.1) the above relation reads:

$$
g(n, n) V(v, r)+[g(U, n)]^{2} \rho(v, r) \geq 0
$$

then we see that $T_{\mu \nu}$ obeys or not the weak energy condition according to the explicit expressions of $\rho$ and $V$. We can say that the $T_{\mu \nu}$ in Eq. (3.1) represents a truly quantum source in two respects: firstly, it encodes the effects of the trace anomaly; secondly, it do not satisfy in general the weak energy condition. However, as we will show later on, our

\footnotetext{
${ }^{3}$ This assumption is lawful because the main equations, (4.5) and 4.6), of the model do not depend on the explicit form of the energy density $\rho$ of Hawking particles. Therefore, the function $\rho$ can assume arbitrarily large and negative values and can include the contribution to the black hole mass decrease of all possible kinds of particles participating in the evaporation
} 
results, not depending on the explicit form of $\rho$ and $V$, hold irrespectively of the energy conditions fulfilled by $T_{\mu \nu}$.

The null energy condition is:

$$
T_{\mu \nu} l^{\mu} l^{\nu} \geq 0
$$

for every light-like future directed vector field $l^{\nu}$, and, in our model, it reads:

$$
[g(l, U)]^{2} \rho(v, r) \geq 0
$$

so that, when $\rho \geq 0$, the weak energy condition can still be violated but the null cannot. Furthermore, it is easy to see that, when $\rho \geq 0, T_{\mu \nu}$ can be thought as the energymomentum tensor of a null scalar field with trace anomaly:

$$
T_{\mu \nu}=\partial_{\mu} \varphi \partial_{\nu} \varphi+g_{\mu \nu} V(\varphi) .
$$

First of all, let us notice that the only properties of $T_{\mu \nu}$ (see below) that we will need are:

$$
T_{r v}^{2}-T_{r r} T_{v v}=\left(g_{r v}\right)^{2} V^{2}, \quad g^{\mu \nu} T_{\mu \nu}=4 V,
$$

and either (3.1) or (3.2) satisfy these relations. Then, when $\rho \geq 0$, the $r-r$ components of both energy momentum tensors (3.1) and (3.2) are greater than zero:

$$
\begin{aligned}
& \rho\left(u_{r}\right)^{2} \geq 0 \\
& \left(\partial_{r} \varphi\right)^{2} \geq 0 .
\end{aligned}
$$

Thus, as far as we are concerned, when $\rho \geq 0$, there is no difference in treating with the $T_{\mu \nu}$ given by Eq. (3.1) or the one in Eq. (3.2).

\section{The Einstein equations}

Up to non radiative terms, that do not contribute to the asymptotic Hawking flux, the Einstein equations $G_{\mu \nu}=\kappa T_{\mu \nu}$ give:

$$
\begin{aligned}
\kappa^{-1} G_{v v} & =V\left(1-\frac{m}{r}\right) \exp (2 j)+\rho\left(u_{v}\right)^{2}, \\
\kappa^{-1} G_{v r} & =-\exp (j) V+\rho u_{r} u_{v}, \quad \kappa^{-1} G_{r r}=\rho\left(u_{r}\right)^{2}, \\
\kappa^{-1} G_{\theta \theta} & =g_{\theta \theta} V, \quad-R=4 \kappa V .
\end{aligned}
$$

The last two equations allow us to write the scalar curvature as:

$$
R=\frac{4}{r^{2}}\left[(m-r) \partial_{r} j+\partial_{r} m\right]
$$

Moreover, thanks to the algebraic properties of $T_{\mu \nu}$ and taking into account that $g(U, U)=$ 0 , we easily get

$$
T_{v r}^{2}-T_{v v} T_{r r}=\left(g_{v r}\right)^{2} V^{2} .
$$

The above relation is a consequence of the non vanishing trace anomaly and it allows to find a closed system of equations for $m$ and $j$ that does not depend on the explicit form of 
$\rho$ and $V$. Indeed, by the field equations, Eq. (4.2) implies the following constraint on the Einstein tensor:

$$
G_{v r}^{2}-G_{v v} G_{r r}=\kappa^{2}\left(g_{v r}\right)^{2} V^{2}=\left(g_{v r}\right)^{2}\left(\frac{R}{4}\right)^{2}
$$

which gives

$$
(m-r)^{2}\left(\partial_{r} j\right)^{2}=-2 r \exp (-j) \partial_{r} j \partial_{v} m
$$

and this relation depends only on the fact that $V \neq 0$, no matter how $\rho$ and $V$ really look like. Then, $\rho$ and $V$ can be positive, negative, and, moreover, can be either local or nonlocal functions, thus representing a truly quantum source, the main equations of the model not depending on their detailed form.

Let us firstly analyse the case $\partial_{r} j=0$. To begin with, thanks to the Einstein equation $G_{r r}=\kappa T_{r r}$, we get

$$
\partial_{r} j=0 \Rightarrow G_{r r}=0 \Rightarrow T_{r r}=0 \Rightarrow u_{r}=0 \Rightarrow U^{v}=0
$$

since it is well known that, if $\rho=0$ (that is, of course, the other solution of $T_{r r}=0$ ) the unique solution is the Reissner-Nordstrom-De Sitter one. Then, via the Bianchi identities, from $U^{v}=0$ it follows that:

$$
L_{U} V=0 \Rightarrow \partial_{r} V=0
$$

where $L_{U}$ is the Lie derivative along $U$. Thus we see that, in this case, $V$ depends only on $v$. From the field equations $G_{\theta \theta}=g_{\theta \theta} V$ and $G_{v r}=\kappa T_{v r}$ we get

$$
V=-\frac{\partial_{r}^{2} m}{2 r}=-\frac{\partial_{r} m}{r^{2}}
$$

The above relations imply that

$$
m=I_{1}(v) r^{3}+I_{2}(v) ; \quad V=-3 I_{1}(v) .
$$

However, this expression, unless $I_{1}(v)=0$, gives a diverging Bondi flux [8] (see below Eq. (5.4)), so that we have to take $I_{1}(v)=0$, that is $V=0$, this yielding a vanishing trace anomaly against the hypothesis $V \neq 0$.

Thus, in order to describe the physical effects of a non vanishing trace anomaly, we have to assume $\partial_{r} j \neq 0$. Eventually, by using $\partial_{r} j \neq 0$ in Eq. (4.4), the equations for $m$ and $j$ reduce to

$$
\begin{gathered}
\frac{\exp (j) \partial_{r} j}{2}=\partial_{v}\left(\frac{r}{m-r}\right) \\
3 m \partial_{r} j=-\exp (-j) 2 r^{2} \partial_{r v}^{2} j+\left(3 r \partial_{r} j-2\right) \partial_{r} m \\
-r \partial_{r}^{2} m-2 r^{2}\left(1-\frac{m}{r}\right)\left(\partial_{r}^{2} j+\left(\partial_{r} j\right)^{2}\right),
\end{gathered}
$$

where it is worth to note that only Eq.(4.5) depends on the assumption $\partial_{r} j \neq 0$. 


\section{The boundary conditions}

We want to describe a transition from two charged blackhole (of the same charge) by the means of the energy-momentum tensor, whose role is to take away energy from the blackhole to infinity. Thus, if the space-time is initially asymptotically flat, so it will be during all the evaporation process. Moreover, the asymptotically flat region is the most important one since we want to evaluate the amount of blackhole's mass that the Hawking particles are able to bring at infinity. For this reason, we are only interested in the boundary conditions in the region where $r \rightarrow \infty$, while, with this toy model, we cannot explore the small $r$-region where quantum-gravitational effects come into play so that we will only consider the region where $r>R^{*}, R^{*}$ being a radius much larger than the Planck scale.

Then, let us look at the conditions to impose on the coefficients of the metric (2.1) that will be assumed to be continuous up to the second derivatives: $m, j \in C^{2}(] 0, T[\times] R^{*}, \infty[)$. First of all, the asymptotic flatness implies that $j$ cannot diverge for $r \rightarrow \infty$, so that $|j| \leq K_{1}<+\infty$. The same is true for $m$. Therefore, either $m$ or $j$ can be expanded there in series of $\frac{1}{r}$ :

$$
j \underset{r \rightarrow \infty}{\sim} \sum_{n \geq 0} \frac{c_{n}(v)}{r^{n}}, \quad m \underset{r \rightarrow \infty}{\sim} \sum_{n \geq 0} \frac{b_{n}(v)}{r^{n}},
$$

where the coefficients $c_{n}(v)$ and $b_{n}(v)$ are bounded continuous functions. Thus, we immediately see that the leading terms of $\partial_{r} j$ and $\partial_{r} m$ in the limit $r \rightarrow+\infty$ (namely, the terms that do contribute to the Bondi mass) fulfil the bounds

$$
\begin{aligned}
& \left|\partial_{r} j\right| \underset{r \rightarrow+\infty}{\lesssim} K_{2} r^{-2} \\
& \left|\partial_{r} m\right| \underset{r \rightarrow+\infty}{\lesssim} K_{2} r^{-2},
\end{aligned}
$$

where $K_{2}$ is a positive constant. Furthermore, the Bondi mass [8] $\Phi_{B}$ of the metric (2.1) is:

$$
\begin{aligned}
\Phi_{B}(v) & =-\frac{1}{8 \pi} \lim _{r \rightarrow \infty} \int_{S_{r}} g^{r v} \Gamma_{v v}^{v} d S \\
& =\frac{1}{8 \pi} \lim _{r \rightarrow \infty} \int_{S_{r}}\left[e^{j}\left[m+2 r(r-m) \partial_{r} j\right]+2 r^{2} \partial_{v} j-r \partial_{r} m\right] d \Omega,
\end{aligned}
$$

where $S_{r}$ is the sphere $\{r=$ const, $v=$ const $\}$. Thus, we see that the previous fall-off conditions ensure a finite Bondi flux provided we also take

$$
\left|\partial_{v} j\right| \underset{r \rightarrow+\infty}{\lesssim} K_{3} r^{-2}
$$

where $K_{3}$ is a positive constant that can be chosen to be equal to $K_{2}$. Moreover, since the total Bondi flux is bounded from above (otherwise the energy conservation would be violated), we have to impose that

$$
\left|\int_{0}^{T} \partial_{v} \Phi_{B}(v) d v\right|<+\infty
$$


from which it follows

$$
\left|\int_{0}^{T}\left(\partial_{r} j\right) d v\right| \underset{r \rightarrow+\infty}{\leq} K_{4} r^{-2}
$$

$K_{4}$ being a positive constant. It is worth to note here that the requirement of a finite total Bondi flux is a necessary condition to ensure the conservation of energy, since the total Bondi flux is nothing but the difference between the final and the initial mass of the blackhole. For these reason, the above relation has to be fulfilled even in the limit $T \rightarrow+\infty$. Moreover, the condition (5.5) imposes a new constraint only on $\partial_{r} j$ while, as far as the other terms are concerned, the conditions (5.2) and (5.3) suffice to satisfy the condition (5.5). This fact suggests that, on physical grounds, the term $\int_{0}^{T}\left(\partial_{r} j\right) d v$ should give the main contribution to the total Bondi flux. It will be a self-consistency test of these computations to confirm this expectation by showing that in the formula of the final mass only the term $\int_{0}^{T}\left(\partial_{r} j\right) d v$ appears explicitly. Then, the problem reduces to solve Eqs. (4.5) and (4.6) with the boundary conditions

$$
\begin{gathered}
\left.\left.m, j \in C^{2}(] 0, T\right] \times\right] R^{*}, \infty[), \\
|m|,|j| \leq K_{1}<+\infty,\left|\partial_{v} j\right|,\left|\partial_{r} m\right|,\left|\partial_{r} j\right| \underset{r \rightarrow+\infty}{\leq} K_{2} r^{-2} \\
\lim _{r \rightarrow \infty}\left|\int_{0}^{T}\left(\partial_{r} j\right) d v\right| \leq K_{4} r^{-2}, \\
m(0, r)=M>0 ; j(0, r)=k_{1} ; m(T, r)=\bar{M} \geq 0 ; j(T, r)=k_{2},
\end{gathered}
$$

where $K_{1}, K_{2}, K_{4}, k_{1}$ and $k_{2}$ are positive constants. It is important to stress that Eqs (4.5) and (4.6), not depending on the explicit form of $\rho$ and $V$, are not coupled with the matter source but, however, because of the relation (4.3), are a direct consequence of the non vanishing trace anomaly.

\section{The non vanishing final mass}

Now, it will be proven that the trace anomaly prevents a complete evaporation. Indeed, by using the boundary conditions $j(0, r)=0$ and $m(0, r)=2 M-\frac{Q^{2}}{r}$, from Eq. (4.5) it follows that the function $m$ can be expressed in closed form in terms of $j$ in the following way:

$$
m(v, r)=r-\frac{r}{\frac{r^{2}}{r^{2}-2 r M+Q^{2}}-\frac{1}{2} \partial_{r} \int_{0}^{v} \exp [j(\tau, r)] d \tau},
$$

whose time derivative reads

$$
\partial_{v} m=-\frac{r e^{j} \partial_{r} j}{2\left(\frac{r^{2}}{r^{2}-2 r M+Q^{2}}-\frac{1}{2} \partial_{r} \int_{0}^{v} \exp [j(\tau, r)] d \tau\right)^{2}} .
$$

Thus, thanks to Eq. (4.1), the sign of $\partial_{v} m$ is directly related to the sign of $\rho$. The final mass $\bar{M}$ may be expressed as

$$
\bar{M}=\frac{1}{2}\left[\frac{Q^{2}}{r}+r-\frac{r}{\frac{r^{2}}{r^{2}-2 r M+Q^{2}}-\frac{1}{2} \partial_{r} \int_{0}^{T} \exp [j(\tau, r)] d \tau}\right] .
$$


It is worth to note that $m(v, r)$, and then $\bar{M}$, cannot be negative. In fact, by using Eqs (5.7) and (6.3) we have:

$$
m \underset{r \rightarrow+\infty}{\sim} r-\frac{r}{1+2 \frac{M}{r}-\frac{Q^{2}}{r^{2}}-\frac{v K_{2} \exp \left[K_{1}\right]}{2 r^{2}}} \geq 0
$$

being $M>0$. Furthermore, by Eq. (6.2), it is trivial to show that:

$$
\begin{aligned}
& \rho \geq 0 \Rightarrow \partial_{r} j \leq 0 \Rightarrow \bar{M} \geq M \\
& \rho<0 \Rightarrow \partial_{r} j>0 \Rightarrow \bar{M}<M .
\end{aligned}
$$

Then, when $\rho \geq 0$, so that $T_{\mu \nu}$ describes a null scalar field violating, at most, the weak energy condition but not the null one, the evaporation does not take place. Moreover, in this case, the boundary conditions (5.7) do not play any role.

However, even when $\rho<0$, a remnant is left. In fact, Eq. (6.3) implies that the function

$$
F(r)=\frac{1}{2} \int_{0}^{T} \exp [j(v, r)] d v
$$

has to satisfy the following ordinary differential equation:

$$
\partial_{r} F=\frac{r^{2}}{r^{2}-2 r M+Q^{2}}-\frac{r^{2}}{r^{2}-2 r \bar{M}+Q^{2}} .
$$

Since the initial blackhole is not extremal, we can write

$$
\frac{r^{2}}{r^{2}-2 r M+Q^{2}}=\frac{1}{r_{+}-r_{-}}\left(\frac{r^{2}}{r-r_{+}}-\frac{r^{2}}{r-r_{-}}\right)
$$

where

$$
r_{ \pm}=M \pm \sqrt{M^{2}-Q^{2}}
$$

Eqs (6.4) and (6.5) give:

$$
F=r+\frac{1}{r_{+}-r_{-}} \ln \frac{\left(r-r_{+}\right)^{r_{+}^{2}}}{\left(r-r_{-}\right)^{r_{-}^{2}}}-\int^{r} \frac{x^{2} d x}{x^{2}-2 x \bar{M}+Q^{2}}+N
$$

where $N$ is an integration constant.

The above relation is not compatible with the boundary conditions. In fact, unless $\bar{M}=M$, it is easy to see that

$$
F(r) \underset{r \rightarrow+\infty}{\rightarrow} \infty,
$$

while the boundary conditions (5.7) imply

$$
\infty>\frac{1}{2} T e^{K_{1}} \geq \frac{1}{2} \int_{0}^{T} e^{j(v, r)} d v=F(r) \underset{r \rightarrow+\infty}{\rightarrow} \infty .
$$

Furthermore, main qualitative features do not change if we also allow a variation of the charge, the result being always incompatible with the formation of a naked singularity. 
Then, even with the "worst" energy-momentum tensor (i.e. a $T_{\mu \nu}$ violating all the energy conditions), the surface gravity cannot vanish preventing the formation of a naked singularity, that is, the evaporation stops because of the trace of the energy-momentum tensor that carries away energy from the blackhole to infinity 16]. Moreover, thanks to the boundary condition (5.8), it is easy to see that the same results also hold in the limit $T \rightarrow \infty$. In fact, Eq. (6.3) shows that, in order to have a final mass different from the initial one, the following relation should hold:

$$
\int_{0}^{\infty} \exp [j(v, r)] \partial_{r} j d v \underset{r \rightarrow+\infty}{\sim} \frac{K_{5}}{r}
$$

$K_{5}$ being a positive constant (otherwise, the coefficient of the $1 / r$-term in the denominator of the r.h.s. of Eq. (6.3), that represents the blackhole mass, would not change). However, from relation (5.8), we get

$$
\int_{0}^{\infty} \exp [j(v, r)] \partial_{r} j d v \leq e^{K_{1}} \frac{K_{4}}{r^{2}}
$$

and the above relation is clearly incompatible with Eq. (6.7). By taking the limit of a vanishing charge [16], we obtain that the trace anomaly of the Hawking particles is able to stop the evaporation irrespectively of the positivity conditions fulfilled by the energymomentum tensor.

In conclusion, in this toy model, it could be possible to take care of the puzzles raised by the Hawking radiation, such as the possible violations of the third law, formation of naked singularities, the unitarity puzzle and the information loss paradox [16], by taking into account the trace anomaly via the exact Einstein equations. This is a quite interesting results since the trace anomaly, that is a generic feature of QFT (namely, it is related to the $\beta$-function of the quantum fields that can vanish only in highly idealized (super)symmetric theories), has a prominent role in stopping the evaporation, so, in some sense, QFT itself could prevent the formation of a naked singularity and the loss of unitarity. This would be a positive self-consistency check for QFT [16]. To complete the analysis, the explicit expression, in terms of the charge $Q=Z e$, of the remnant mass $\bar{M}=M_{P} f(Z)$ should be determined, where $M_{P}=\sqrt{\hbar c / G}$ denotes the Planck mass. At this level of analysis, in which numeric computations have not yet been performed, we may only say that $f(0) \neq 0$, since previous results also hold in the limit $Q \longrightarrow 0$. The function $f$ strongly depends on the conformal anomaly whose expression, being determined by all quantum fields that contribute to the Hawking radiation, is not available yet. However, the remnant mass is an increasing function of conformal anomaly because this latter is responsible for stopping the evaporation.

Many previous works on this topic [3], [29] showed that, by neglecting the fourdimensional trace anomaly, the blackhole, in a time of the order $O\left(M^{3}\right)$, can loose all its mass, as first predicted by Hawking [22]. Thus, the following questions arises:

which is the new insight related to the four dimensional trace anomaly?

when is the trace anomaly not negligible anymore? 


\section{Field-theoretical features}

In this section the Planck constant $\hbar$, the light velocity $c$ and the gravitational constant $G$ have been restored. It is easy to check that Eqs (4.5) and (4.6) are formally scale-invariant. Namely, if $m_{0}$ and $j_{0}$ are a solution of the system, then $\lambda m_{0}$ and $j_{0}$ are a solution too (provided we also make the transformation $r \rightarrow \lambda r, t \rightarrow \lambda t$ ). Then, because of the freedom in changing the energy scale, Eqs (4.5) and (4.6) by themselves do not provide us with a lower bound on the remnant mass.

However, this is only formally true. For instance, the presence of a nonvanishing cosmological constant $\Lambda$, being the value of $\Lambda$ fixed by experiments, would violate the scale invariance because no scale transformation on $\Lambda$ could be performed. In other words, $\Lambda$ introduces a characteristic energy scale in the evaporation process. The same is true for the trace anomaly, because it also breaks the scale invariance and this breaking is hidden in the r.h.s. of the Einstein equations.

Now we will present a highly qualitative argument to obtain a lower bound for the remnant mass and a new insight into the relations between a complete evaporation and the loss of unitarity on purely thermodynamical grounds, although, in order to know exactly when the trace anomaly comes into play, one should have the explicit expressions of both $\rho$ and $V$ or, at least, an estimate of their ratio $V / \rho$. Furthermore, in order to provide a clear physical intuition, we will follow as close as possible an interesting analogy with the computation of the Casimir force. In the following, for the sake of simplicity, we will consider the case of a Schwarzschild blackhole shortly analysed in [16], although the analysis can be extended to the charged case too. The treatment is absolutely non rigorous since it is intended only to give an intuitive picture of the process.

As originally found by Hawking [22], the expectation value of the operator number of a bosonic field of spin zero, measured by a static observer in the asymptotic future of Schwarzschild blackhole of mass $M$, is

$$
n_{i}=\frac{1}{\exp \left(\hbar \omega_{i} / k T_{H}\right)-1},
$$

where $k$ is the Boltzmann constant and

$$
T_{H}=\frac{\hbar c^{3}}{8 \pi k G M}
$$

is the Hawking temperature. Since our purpose is to get only an order of magnitude estimate, in the following the greybody factors will be neglected.

It is known that the basic ingredients to get the relation (7.1) are the existence of a horizon and the fact that on curved space-times, because of the lacking of a preferred Poincaré symmetry group, the notion of particle is not an invariant concept. Thus, since the Einstein equations are not needed to get the above relation, it is lawful to say that the Hawking effect is only "kinematical" [37]. From the physical point of view, this means that, since in deducing relation (7.1) the background metric is kept fixed, we are neglecting the backreaction effects of the emitted particles on the metric so that the energy conservation 
is not fulfilled. In fact, the blackhole should give to the Hawking particles the energy they need to be created but, if $M$ does not change, this, obviously, does not happen.

Hence, by imposing the conservation of energy, we will take into account the backreaction, i.e. we will ensure that this thermal particles are created by the mass lost by the blackhole. In the following, we will assume that the expectation value of the operator number $n_{i}=n_{i}(M)$ changes adiabatically as a function of $M$. This assumption is well verified in all the situations in which the blackhole thermodynamics is applicable [29].

Now we will compute how the expectation number (7.1) increases due to the decrease of the blackhole mass and then we will write the conservation of energy for this process. This point of view is quite similar to the one adopted in the computation of the Casimir force. In fact, in the latter case, one first computes the zero modes and the vacuum energy (that is, of course, a divergent but unobservable quantity) in a configuration with some nontrivial boundaries, and then computes the variation of the vacuum energy due to an infinitesimal change in the boundaries that is finite and observable as well. In the blackhole case, the boundary is the Schwarzschild radius so that a change in the mass can be thought as a change in the boundary. For this reason, we should do the same and obtain the same results (namely, that the energy due to the particles created by the mass lost by the blackhole is a finite quantity). Therefore, if $M \rightarrow M+\delta M$, then $n_{i} \rightarrow n_{i}+\delta n_{i}$ with

$$
\delta n_{i}=\frac{\partial n_{i}}{\partial M} \delta M=-\frac{8 \pi G \hbar \omega_{i} \exp \left(\hbar \omega_{i} / k T_{H}\right)}{\hbar c^{3}\left(\exp \left(\hbar \omega_{i} / k T_{H}\right)-1\right)^{2}} \delta M .
$$

Then, the main step to get a lower bound of the remnant mass is to impose that the new particles are created by the mass lost by the black-hole, that is, we have to fulfil the conservation of energy:

$$
c^{2} \delta M+\sum_{i} \hbar \omega_{i} \delta n_{i} \leq 0
$$

where, since the blackhole is evaporating, $\delta M<0$ and in Eq. (7.3) the inequality has to be used. In fact, besides the spin-zero bosons described by the relation (7.1), the mass lost by the blackhole also gives rise to other kind of particles, such as fermions or bosons with higher spin. For this reason, if we would use the equality in Eq. (7.3), we would neglect all the other particles that the blackhole is able to create. Then, the second term in Eq. (7.3) encodes, in the thermodynamical limit, the backreaction effects of the emitted particles on the gravitational field. It is clear from Eq. (7.3) that, as long as $M$ is big enough, the second term in the inequality is completely negligible and the usual blackhole thermodynamics should apply.

However, the smaller is $M$, the harder is to fulfil Eq. (7.3). In fact, from Eqs. (7.2) and Eq. (7.3) it follows that

$$
1 \geq \frac{8 \pi}{\left(c^{2} M_{P}\right)^{2}} \sum_{i} \frac{\left(\hbar \omega_{i}\right)^{2} \exp \left(\hbar \omega_{i} / k T_{H}\right)}{\left(\exp \left(\hbar \omega_{i} / k T_{H}\right)-1\right)^{2}}, .
$$

It is worth to note here that, in the above relation, as expected on the grounds of the analogy with the Casimir case, neither infrared nor ultraviolet divergences appear, so that we do not need any regularization. Furthermore, Eq. (7.4) implies that the conservation 
of energy can be satisfied only for $M \geq \bar{M}$, where $\bar{M}$ is implicitly defined by the following equation:

$$
1=\frac{8 \pi}{\left(c^{2} M_{P}\right)^{2}} \sum_{i} \frac{\left(\hbar \omega_{i}\right)^{2} \exp \left(8 \pi G \omega_{i} \bar{M} / c^{3}\right)}{\left(\exp \left(8 \pi G \omega_{i} \bar{M} / c^{3}\right)-1\right)^{2}},
$$

It is worth noting here that this is only a lower bound and that the remnant could be quite larger than the Planck mass. In fact, if we would be able to include in Eq. (7.3) all the different kinds of massless particles that contribute to the evaporation process (such as fermions or bosons with higher spin, etc.), then the inequality in Eq. (7.3) would become an equality, so that there would not be phase space available anymore for the evaporation itself. Moreover, as it has been shown above, if the trace anomaly $V$ was of the same order of $\rho$ then the evaporation would halt well before reaching the Planck scale.

Thus, the conservation of energy, and then the backreaction, prevents the blackhole mass $M$ from being lower than $\bar{M}$. Since a smaller mass is not allowed by the energy conservation, the remnant is stable. Besides being an interesting result in itself, this makes clear the relation between energy conservation and complete evaporation. In fact, it has been speculated [23] that, since it seems that a complete evaporation cannot be ruled out, QFT has to be generalized to allow nonunitary processes. However, if one tries to do this, then the energy conservation is lost [20], [4]. Instead, Eq. (7.4) shows that the energy conservation itself stops the evaporation. Moreover, this conclusion fits very well with the results obtained in [13], [1].

\section{Conclusions and perspectives}

In this paper a toy model is proposed to describe a transition from a Reissner-Nordstrom blackhole of mass $M$ and charge $Q$ to a Reissner-Nordstrom blackhole of mass $\bar{M}$ and charge $Q$. The basic equations are derived from the non-vacuum Einstein equations, the source being an energy-momentum tensor with a non vanishing trace representing the massless quanta of the Hawking particles. Nevertheless, the results do not depend on the explicit form of the energy-momentum tensor, thus allowing in principle arbitrary violations of all the energy conditions. We showed, via the exact Einstein equations, that the trace of the energy-momentum tensor prevents any lowering of the surface gravity saving, in this way, the third law of blackhole thermodynamics from the Hawking radiation and preventing the formation of a naked singularity. In the limit of a vanishing charge, this approach shows that the trace anomaly of the Hawking particles stops the evaporation. In this way, the information loss paradox could be resolved by taking into account the information detained by the remnant and by the Hawking radiation through the Bekenstein mechanism [6]. We also briefly analyzed the field-theoretical connection between the blackhole evaporation and the cosmological constant.

\section{Acknowledgments}

The authors wish to thank D.Grumiller, G.Esposito and S.Sonego for remarks and important bibliographic suggestions. 


\section{References}

[1] Y. Aharonov, A. Casher, S. Nussinov, Phys. Lett. B 191 (1987) 51;

[2] J. Bardeen, B. Carter, S. W. Hawking, Comm. Math. Phys. 31 (1973) 161;

[3] J. Bardeen, Phys. Rev. Lett. 46 (1981) 382;

[4] T. Banks, M. E. Peskin, L. Susskind, Nucl. Phys.B 244 (1984) 125;

[5] J. D. Bekenstein, Phys. Rev. D 7 (1973) 2333;

[6] J. D. Bekenstein, Phys. Rev. Lett. 70 (1993) 3680;

[7] A.O. Barvinsky, Yu. V. Gusev, G. A. Vilkovisky, V.V Zhytnikov, J. Math. Phys. 35 (1994) 3543

[8] H. Bondi, M.G. J. Van der Burg, A. W. K. Metzner, Proc. Roy. Soc. A269, (1962) 21;

[9] A. Bonanno, M. Reuter, Phys. Rev. D 62 (2000) 043008 and references therein;

[10] M. Bojowald, Phys. Rev. Lett. 86 (2001) 5227;

[11] M. Bojowald, Phys. Rev. D 64 (2001) 084018;

[12] P. R. Brady, I. G. Moss, R. C. Myers, Phys. Rev. Lett. 80 (1998) 3432;

[13] R. Casadio, Phys.Lett. B 511 (2001) 285;

[14] S. Chandrasekhar, J. Hartle, Proc. Roy. Soc. Lond. A384 (1982) 301;

[15] D. Christodoulou, Ann. of Math. 104, n. 3 (1994) 607;

[16] F. Canfora, G. Vilasi, Trace anomaly and black holes evaporation, gr-qc/0302036;

[17] M. Dafermos, gr-qc/0209052;

[18] J. R. David, G.Mandal, S. R.Wadia, Phys. Rept. 369 (2002) 549;

[19] R. Dijkgraaf, H. Verlinde, E. Verlinde, Nucl. Phys. B 371 (1992) 269;

[20] J. Ellis, J. Hagelin, D. V. Nanopoulos, M. Srednicki, Nucl. Phys.B 241 (1984) 381;

[21] D. Grumiller, W. Kummer, D. V. Vassilevich, Phys. Rept. 369 (2002) 327 and references therein;

[22] S. W. Hawking, Nature (London) 243 (1974) 30;

[23] S. W. Hawking, Phys. Rev. D 14 (1976) 2460;

[24] W. Israel, Phys. Rev. Lett. 57 (1986) 397;

[25] I. Jack, D. R. T. Jones, J. Panvel, Nucl. Phys. B 393 (1993) 95;

[26] P. S. Joshi, gr-qc/0206087 and references therein;

[27] D. I. Kazakov, S. N. Solodukhin Nucl. Phys. B 429 (1994) 153;

[28] S. P. Martin, Nucl. Phys. B 327 (1989) 178;

[29] S. Massar, Phys. Rev. D 52 (1995) 5861 and references therein;

[30] S. Massar, R. Parentani, Nucl. Phys. B 575 (2000) 333;

[31] V. Mukhanov, A. Wip, A. Zelnikov, Phys. Lett. B 332 (1994) 283; 
[32] S. Nojiri, S. Odintsov, Int. J. Mod. Phys. A16 (2001) 1015 and references therein;

[33] D. N. Page, Phys. Rev. Lett. 44 (1980) 301;

[34] D. N. Page, Phys. Rev. D 25 (1982) 1499;

[35] R. Parentani, T. Piran, Phys. Rev. Lett. 73 (1994)2805;

[36] T. Thiemann, gr-qc/0110034 and references therein;

[37] M. Visser, Phys. Rev. Lett. 80 (1998) 3436 and references therein;

[38] E. Witten, Nucl. Phys. B 311 (1988) 46;

[39] E. Witten, Nucl. Phys. B 323 (1989) 113;

[40] J. W. York, Phys. Rev. D 31 (1985) 775; 